\title{
Soliton Pulse Model in Different Order for Communication Compatibility
}

\author{
Muhammad Sufi, Azam Mohamad, Saktioto, and Jalil Ali
}

\begin{abstract}
In this paper the variation in soliton pulse with different order number, $\mathrm{N}$ has been investigated and analyzed from Nonlinear Schrodinger Equation (NLSE). The Split-Step Method has used for numerical calculation to form soliton pulse model. The change in pulse shape and variation in power level have been observed with the increase in the soliton pulse order number. Thus, the soliton pulse parameters can be optimized to acquire the desired output. The dispersion of the pulse is ascertained for observing the pulse compression phenomena.
\end{abstract}

Index Terms-Soliton pulse, order (N), power.

\section{INTRODUCTION}

Time division multiplexing (TDM) in fiber optics is going advanced by day. By using a half-soliton period length of low-loss single-mode fiber, we have been able to demonstrate the pulse compression and pulse splitting associated with several higher-order soliton as well as to observe the fundamental soliton [1]. The speed of data transfer that up to $640 \mathrm{Gbit} / \mathrm{sec}$ [2] is main advantage of TDM that can increase the capacity of transmission. On the other hand, TDM can perform multiple access system as well as the number of star fiber coupler [3]. Soliton wave is useful for long haul transmission where within TDM system with soliton control, it enables sending an ultrahigh-speed signal channel transmission over 10, $000 \mathrm{~km} \mathrm{[4].} \mathrm{Research}$ has pointed out numerically that a stable, steady-state, nonlinear pulse can exist even in the average normal the group velocity dispersion (GVD) region [5]. The transmission speed of $80 \mathrm{~Gb} / \mathrm{s}$ has been increased to 160 $\mathrm{Gb} / \mathrm{s}$ using polarization and time-domain multiplexing techniques [6]. Soliton communication system with a novel scheme of channel multi/demultiplexing where the proposed system is a multi-energy-level system which uses soliton with different energies each of which corresponds to a data channel [7].

In this study, the Nonlinear Schrodinger Equation is solved numerically. The Nonlinear Schrodinger (NLS) equation includes only the GVD and the self-phase modulation (SPM) well known in the fiber, and it construct

M. Sufi, A. Mohamad, Saktioto, and J. Ali are with Institute of Advanced Photonics Science, Nanotechnology Research Alliance, University Technology Malaysia, 81310 Johor Bahru, Malaysia(email:zzzsopi@gmail.com, jalilali@utm.my).

Saktioto is with Physics Department, University of Riau, Pekanbaru, Indonesia(email: saktioto@yahoo.com). bright and dark soliton-type pulse propagation in anomalous and normal dispersion regimes, respectively [8]. The order of soliton pulse, $\mathrm{N}$ is analyzed to perform the soliton pulse shape that stable for communication. This equation governs the general situation of dispersion propagation of a pulse envelope with a high carrier frequency in a weakly nonlinear medium. Although the NLS equation includes only two physical effects, GVD and SPM, it describes a variety of nonlinear optical phenomena. Depending on the relative signs of linear group-velocity dispersion and nonlinearity induced self-phase modulation, they combine to allow bright soliton [9], modulation instability [10] and dark soliton [11]. The analytical treatment of NLSE is for determine the change of power for different order of soliton.

\section{THEORETICAL CONSIDERATION}

Soliton wave equation is governed by Nonlinear Schrodinger Equation (NLSE) from the partial differential equation of hyperbolic equation as given by [12]:

$$
i \frac{\partial A}{\partial z}=\frac{\beta_{2}}{2} \frac{\partial^{2} A}{\partial T^{2}}-\gamma|A|^{2} A
$$

By using some of the new parameter $u=\frac{A}{\sqrt{P_{0}}} \xi=\frac{z}{L_{D}}$, and $\tau=\frac{T}{T_{0}}$ that will normalize the Eq. (1) to be:

$$
i \frac{\partial u}{\partial \xi} \frac{1}{L_{D}}=\frac{\beta_{2}}{2 T_{0}^{2}} \frac{\partial^{2} u}{\partial \tau^{2}}-\gamma|u|^{2} u P_{0}
$$

By introducing the dispersion length, $L_{D}=\frac{T_{0}^{2}}{\left|\beta_{2}\right|}$ and nonlinear length $L_{N L}=\frac{1}{\gamma P_{0}}$ and inserting $L_{D}$ and $L_{N L}$ into Eq. (2), it will yield,

$$
i \frac{\partial u}{\partial \xi}=\frac{\beta_{2}}{2} \frac{\partial^{2} u}{\partial \tau^{2}}-N^{2}|u|^{2} u
$$

where new parameter, $\mathrm{N}$ as the order of soliton is introduced $N^{2}=\frac{\gamma P_{0} T_{0}^{2}}{\left|\beta_{2}\right|}$

By solution of profiles $u(\xi, \tau)$ take the forms of

$$
u(\xi, \tau)=\operatorname{sech}(\tau) e^{-\frac{i}{2} C \tau^{2}}
$$


where $\mathrm{C}$ is the chirp parameter caused by SPM and GVD that balanced each other as soliton propagate thus maintain the shape of soliton pulse as it travels.

It is determined that $N^{2}=\frac{L_{D}}{L_{N L}}$, so from that equation we can understand that the change of order, $\mathrm{N}$ is proportional with the change of square root of dispersion length and inversely with the nonlinear length. In this study, the order of soliton, $\mathrm{N}$ is modified, so literally the increase of $\mathrm{N}$ will also increase the dispersion length of the fiber if we consider the nonlinear remain constant. The vast change of dispersion length will lead to pulse broaden and the pulse will lose power within it when the amplitude of the pulse shrinkage from the equation $\mathrm{P}=|\mathrm{A}|^{2}$.

The numerical calculation of soliton pulse by using SplitStep method is shown in next chapter where the result is focused on the change of the order of soliton, $N$. If the soliton order is unity $(\mathrm{N}=1)$, the soliton wave propagates unchanged along the proposed transmission line [13], this show up the propagation of fundamental soliton pulse. The interplay between the anomalous GVD and the SPM effects leads to soliton pulse generation. If $\mathrm{N}$ is less than unity value or high dispersive regime, the GVD affect dominant to the SPM effect and causes pulse broadening. In the case of $\mathrm{N}>1$ or high nonlinearity regime, the SPM effect is dominant that leads to periodic pulse shape changing [12] so that the pulse is compressed along certain length of the proposed transmission line. Self-phase modulation (due to the nonlinearity) tends to lower frequencies in the leading half of the pulse and to raise those in the trailing half. When acted upon by negative dispersion, such a chirp produces pulse compression. In the correct conditions, such compression can be substantial and represents one of the many possible practical consequences of soliton phenomena [1].

\section{RESUlTS AND Discussion}

Based on the numerical calculation of the soliton pulse by solving the NLSE, the order of the soliton can be calculated theoretically by using the approximation of $N^{2}=\frac{L_{D}}{L_{N L}}=$ $\frac{\gamma P_{0} T_{0}^{2}}{\left|\beta_{2}\right|}$ where $\mathrm{N}$ standpoint for the order of soliton. By this approximation, we can calculate the variable of soliton order from the minimal order $\mathrm{N}=1$ to the higher order $\mathrm{N}>5$. The result of the first three order of soliton pulse wave from Eq. (4) is shown in Fig. 1 below

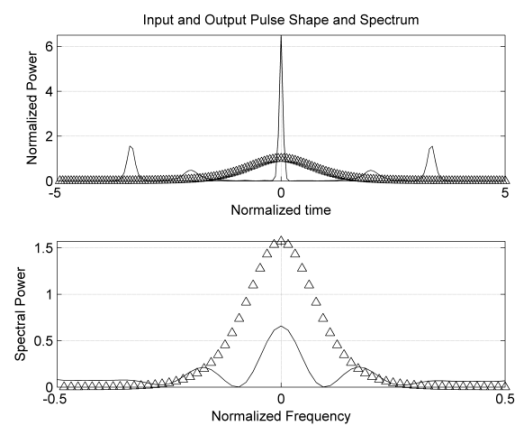

(a)
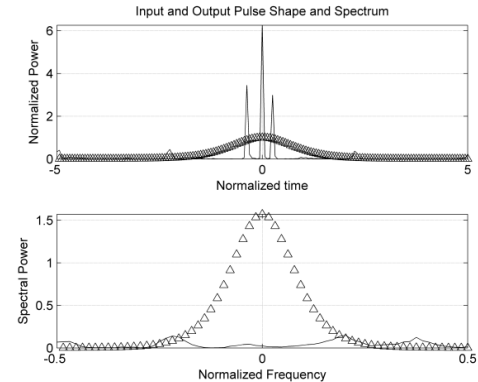

(b)

Fig. 1. Pulse shape at of soliton at (a) first order, (b) third order (the $\Delta$ point for input and the lines point for output).

Results show that the pulse of the soliton wave will follow the number of order of the soliton, N. Each order will bring up the number pulse of soliton wave. For $\mathrm{N}=1$ in Fig. 1 (a) the result is single pulse of soliton and for $\mathrm{N}=2$, the result is double pulse, and triple for $\mathrm{N}=3$, (as shown in Fig. 1 (b)). This indicates that the number of order will acquire the number of pulse. For $\mathrm{N}=1$ both SPM and GVD adjoin in such a way that the SPM-induced chirp is just right to cancel the GVD-induced broadening of the pulse make the pulse seeing stable as it stand.

The normalized power obtained for the first three orders is considered high where it exceeds 6 watts shows that the first three order of soliton pulse has the stable pulse shape. The output power attained is same as the input power of the pulse. With this high power, the pulse can travel in long trip.

For the next higher order of soliton, the characteristic of the pulse is different. The pulse of soliton at $\mathrm{N}=4$ and $\mathrm{N}=5$, the power remains low where it only yields amount around 4 watts .For both pulse, almost same amount of power were formed. However, the pulse shape shows that it is unstable for the balance of between SPM and GVD that govern the chirp of the pulse. The chirp of the pulse is not balance between the tail and the head of the pulse .The result of soliton pulse for order $\mathrm{N}=4$ and $\mathrm{N}=5$ is shown in Fig. 2 (spectrum of the pulse is at the left one).

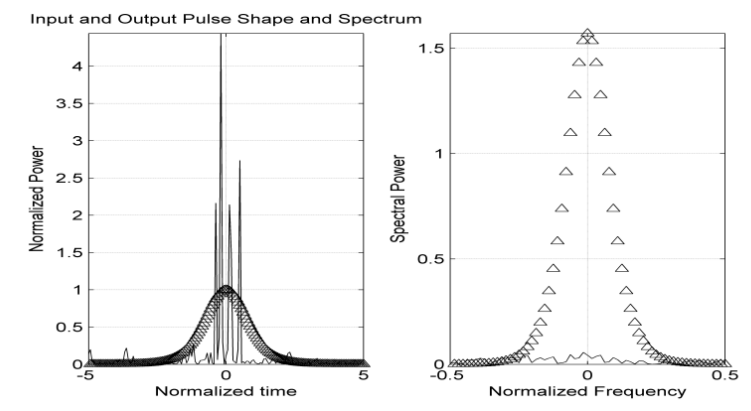

Fig. 2. Soliton pulse shape of order $\mathrm{N}=5$.

For the higher order of soliton, $\mathrm{N}>5$, the shape of soliton become more chaotic and unstable. The formation of soliton pulse is chaotic and not balanced between GVD and SPM. Thus chirping phenomenon (frequency variation on the right side of Fig.2) is generated due to SPM, which leads to the spectral broadening of the pulse without any change in temporal distribution. In the wavelength region where chromatic dispersion is positive, the red-shifted leading edge of the pulse travels slower and moves toward the middle of pulse. Similarly, the blue shifted trailing edge 
travels faster, and also moves toward the middle of the pulse. In this situation SPM causes the pulses to contract. The pulse width becomes narrower and too much chirping is produced by the pulse. The GVD of a signal is function of wavelength, therefore each spectral component can be assumed to travel independently and to undergo a group delay, which ultimately results in pulse broadening [12]. If this condition continues within long haul transmission, the pulse broadening effect will tend the pulse to have interference with each other thus make the detection of the signal become harder and complicated. The pulse signals received have some distortion. The power produced also remains low where it yields around 2 watts only for higher order of soliton pulse $(\mathrm{N}>5)$. Therefore, for the higher order of soliton, the pulse is not suitable to be used in the fiber communication for certain loss may lead to bring the pulse to vanish during the transmission.

\section{SUMMARY}

A new modification of soliton model for higher order has been successfully investigated. The result has shown that the stable pulse profile for fiber communication is within the order of soliton $1 \leq \mathrm{N} \leq 3$ where the power conceded by the pulse keep maintains as the pulse travel. The shape of the soliton pulse also preserved as the SPM and GVD effect sensible with the propagation in anomalous region. It is also shown that the higher order of soliton has the effects on the power level. The power levels decreased as the order of soliton increase. The higher order of soliton pulse $(\mathrm{N}>5)$ also initiated the pulse to narrow down and the chirping becomes chaos. This will cause the information carrying capacity reduced and after a certain amount of overlap by GVD, adjacent pulses cannot be identified at the receiver and error will take place. Thus the selection of soliton order is important to make sure that the soliton pulse can propagate without loss of power in long distance transmission and the information retrieval made up easily.

\section{ACKNOWLEDGEMENTS}

We would like to thank the Institute of Advanced Photonics Science, Nanotechnology Research Alliance, Universiti Teknologi Malaysia. This research work has been financially supported by the Ministry of Higher Education (MOHE) Malaysia with FRGS-Grant Vote No. 78676 and also thank to Physics Dept. University of Riau, Indonesia for generous support of this research.

\section{REFERENCES}

[1] L. F. Mollenauer, R. H. Stolen, and J. P. Gordon, "Experimental observation of picosecond pulse narrowing and solitons in optical fibers," Phys. Rev. Lett. vol. 45, no. 13, pp.1095, 1980.

[2] T. Yamamoto, E. Yoshida, and M. Nakazawa, "640-Gbit/s Optical TDM Transmission Over 92 kmThrough a Dispersion-Managed Fiber Consisting of Single-Mode Fiber and Reverse Dispersion Fiber," IEEE Photonics Technology Letters, vol. 12, no. 3, pp. 353-355, 2000

[3] J. G. Zhang, "Performance of fibre-optic time-division multiple access systems," Electronics Letters, vol. 30, no. 1, pp.66-68, 1993.

[4] M. Nakazawa, H. Kubota, K. Suzuki, E. Yamada, and A. Sahara, "Ultrahigh-Speed Long-Distance TDM and WDM Soliton Transmission Technologies," IEEE Quantum Electronics Journals, vol. 6, no. 2, pp. 363- 396, 2000.
[5] J. H. B. Nijhof, N. J. Doran, W. Forysiak, and F. M. Knox, "Stable soliton-like propagation in dispersion managed systems with net anomalous, zero, and normal dispersion," Electron. Lett. vol. 33, pp. 1063, 1997.

[6] S. G. Evangelides Jr., L. F. Mollenauer, J. P. Gordon, and N. S. Bergano, "Polarization multiplexing with solitons," IEEE, J. Lightwave Technol. vol. 10, no. 1, pp. 28-35, 1992.

[7] H. Hatami-Hanza, A. Mostofi, and P. L. Chu, "A multilevel soliton communication system," J. LightwaveTechnol. vol 15. no. 1 pp. 6-19, 1997.

[8] A. Hasegawa and F. Tappert, "Transmission of stationary nonlinear optical pulses in dispersive dielectric fibers. I. Anomalous dispersion," Appl. Phys. Lett. vol. 23, no. 3, pp.142-144, 1973.

[9] V. E. Zakharov and A. B. Shabat, "Exact theory of two-dimensional self-focusing and one-dimensional self-modulation of waves in nonlinear media," Sov. Phys. JETP, vol. 34, pp. 62-69, 1972.

[10] K. Tai, A. Hasegawa, and A. Tomita, "Observation of modulational instability in optical fibers," Phys. Rev. Lett. vol. 56,no. 2, pp. 135138, Jan. 1986.

[11] D. Krokel, N. J. Halas, D. Grischkowsky, and G. Giuliani, "Dark Pulse Propagation in Optical Fibers," Phys. Rev. Lett. vol. 60, pp. 2932, 1988.

[12] G. P. Agrawal, Nonlinear Fiber Optics, San Diego, C A, Academic Press, Inc. 3rd Edition, 2001.

[13] Gharakhili, Shahabadi, and Hakkak, "Bright and dark soliton generation in a left-handed nonlinear transmission line with series nonlinear capacitors," Progress In Electromagnetics Research, PIER 96, pp. 237-249, 2009.

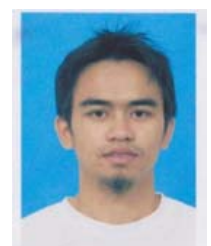

M. S. Roslan born at Kulai, Johor, Malaysia on $22^{\text {nd }}$ November 1988. He received his B.Sc. degree of Science (Physics) in 2010 at Universiti Technologi Malaysia (UTM) at Johor Bahru,Malaysia. At present he studies his master's degree under Institute of Advanced Photonics Science, Nanotechnology Research Alliance, UTM. His research theme is on soliton wave propagation and optical logic gates in micro ring resonators under the supervision of Prof. Dr. P. P. Yupapin (KMITL), Prof. Dr. Jalil Ali (UTM) and Dr. Saktioto (UTM) and now he is one of the members of IAENG.

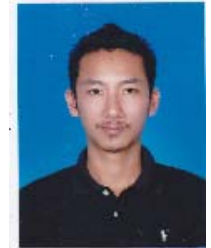

A. Mohamad born at Pontian, Johor, Malaysia on $25^{\text {th }}$ May 1988. He received his B.Sc. degree of Science (Physics) in 2010 at Universiti Technologi Malaysia (UTM) at Johor Bahru,Malaysia. At present he studies his master's degree under Institute of Advanced Photonics Science, Nanotechnology Research Alliance, UTM. His research theme is on electro-optic tuning based on micro ring fiber under the supervision of Prof. Dr. P. P. Yupapin (KMITL), Prof. Dr. Jalil Ali (UTM) and Dr. Saktioto (UTM) and now he is one of the members of IAENG.

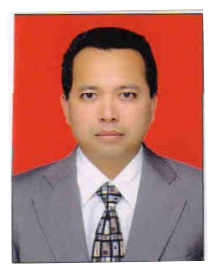

Saktioto born at Bagansiapiapi, Indonesia on $30^{\text {th }}$ October 1970. He received his $\mathrm{PhD}$ in plasma physics from Universiti Teknologi Malaysia (UTM), Johor Bahru Malaysia on 2008 on Optoelectonic and Integrated Optics. His research areas are on plasma physics and optical computing. He is currently the Head of Nano-photonics Research Group, UTM.

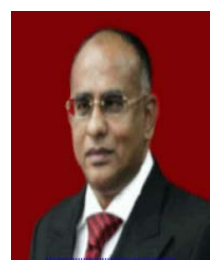

Jalil Ali born at Johor Bahru, Johor on $2^{\text {nd }}$ April 1959 received his $\mathrm{PhD}$ in plasma physics from Universiti Teknologi Malaysia (UTM), Malaysia in 1990. At present, he is a Professor of Photonics at the Institute of Advanced Photonics Science, Nanotechnology Research Alliance and the Physics Department, UTM. From 1987-2010, he has held numerous faculty and research positions including the Dean/Director, Bureau of Innovation and Consultancy. He was instrumental in establishing and forging University-Industry collaboration in Malaysia. He has authored/coauthored more than 300 technical papers published in international journal, three books and a number of book chapters. His areas of interests are in photonics, optical solitons, fiber couplers, nanowaveguides and plasma focus devices. 